\title{
Lesión medular y uso de sustancias: una revisión sistemática
}

\section{Spinal cord injury and substance use: a systematic review}

\section{P. Lusilla-Palacios*, Carmina Castellano-Tejedor**}

*Departamento de Psiquiatría, Hospital Universitario Vall d'Hebron, CIBERSAM, Universidad Autónoma de Barcelona, Passeig de la Vall d'Hebron 119-129, 08035 Barcelona, España

**Instituto de Investigación Vall d'Hebron, Passeig de la Vall d'Hebron 119-129, 08035 Barcelona, España

\section{Resumen}

El objetivo de este estudio fue el de revisar los hallazgos recientes sobre la prevalencia de uso de sustancias (US) y trastornos por uso de sustancias (TUS) y discutir su impacto en la salud en población con lesiones medulares (LM). Para este propósito, se realizaron búsquedas asistidas por ordenador en MEDLINE (PubMed) y en la Biblioteca Cochrane. A partir de un conjunto inicial de 59 artículos, 52 cumplieron los criterios de inclusión. La mayoría de trabajos se centraban en el consumo de alcohol y tabaco, y sólo unos pocos informaron acerca del uso de otras sustancias. El tipo de diseño de investigación fue mayoritariamente transversal y descriptivo, siendo escasos los estudios de intervención y longitudinales. A pesar de la alta prevalencia de US documentada en pacientes con LM, apenas existen trabajos sobre el consumo previo a la LM y de diseño longitudinal. Además, cuando se ha evaluado el TUS, éste no se ha estudiado de forma sistemática siguiendo criterios diagnósticos CIE o DSM. El alcohol ha resultado ser la sustancia más consumida entre esta población. Además, los pacientes con US han mostrado peores puntuaciones en distintos indicadores de salud. Por ello, se necesita más investigación para seguir avanzando en este ámbito de estudio, así como para poder diseñar intervenciones preventivas más efectivas adaptadas a las necesidades específicas de esta población, y para sugerir prioridades de investigación.

Palabras clave: Lesión medular, uso de sustancias, trastorno por uso de sustancias, comportamiento adictivo, salud.

Abreviaturas: LM: Lesión Medular(es); TUS: Trastorno por Uso de Sustancias; US: Uso de Sustancias

\begin{abstract}
The objective of this study was to review recent findings about the prevalence of substance use (SU) and substance use disorders (SUD), and to discuss the related impact on health in spinal cord injury (SCI) population. For this purpose, computer-aided searches of MEDLINE (PubMed) and the Cochrane Library were conducted. From an initial pool of 59 articles, 52 met the inclusion criteria. Most of the studies referred to alcohol and tobacco and only a few studies reported on other substances. Study designs were mainly cross-sectional and descriptive, with scarce intervention and longitudinal studies. Although a high prevalence of post-injury SU has been documented among SCI patients, limited research exists on pre-injury SU and on longitudinal studies. Moreover, when exploring SUD, it has not been systematically studied in accordance with CIE or DSM criteria. Alcohol appears to be the most consumed substance among this population. Additionally, those patients with SU have shown poorer outcomes in different health indicators. Therefore, more insight is required to increase scientific knowledge in this field and to recommend tailored preventive interventions and research priorities in relation to this population.

Key Words: Spinal cord injury, substance use, substance use disorders, addictive behaviours, health outcomes.

Abbreviations: SCI: Spinal Cord Injury(ies); SU: Substance Use; SUD: Substance Use Disorder(s)
\end{abstract}

Recibido: Septiembre 2014; Aceptado: Abril 2015

Enviar correspondencia a:

Carmina Castellano-Tejedor, Hospital Universitario Vall d'Hebron, Passeig de la Vall d'Hebron 119-129, 08035 Barcelona, España.

E-mail: ccastellano@vhebron.net 
$\mathrm{E}$ 1 impacto de una Lesión Medular (en adelante LM) después de un trauma puede resultar en respuestas emocionales negativas (p. ej., depresión, ansiedad), afrontamiento de desvinculación (p. ej., negación de los factores relacionados con la lesión, evitación) y un funcionamiento psicosocial alterado (p. ej., los roles familiares, la identidad) (Antonak y Livneh, 1991; Hammell, 1992; Vash y Crewe, 2004; Wright, 1960). A veces, la angustia psicológica después de la lesión puede ser tan intensa e insoportable que los pacientes busquen alivio a través del uso de sustancias (Smedema y Ebener, 2010; Treischmann, 1988). Por un lado, las personas que ya son Usuarias de Sustancias antes de sufrir la lesión (en adelante US) pueden agravar su consumo e incrementar las probabilidades de desarrollar un Trastorno por Uso de Sustancias (en adelante TUS) o, en el mejor de los casos, pueden decidir dejar, o reducir, su comportamiento como US (Bombardier, Stroud, Esselman y Rimmele, 2004; Burns y Ditunno, 2001; Krause, 2004; Saunders y Krause, 2011; Smedema y Ebener, 2010).

Los estudios científicos acuerdan que el US, y en particular los TUS, tiene un impacto negativo sobre el trabajo, el rendimiento académico, la vida familiar y social, y también en los estados de salud tanto físico como psicológico; si los TUS ocurren en poblaciones con condiciones de salud severos y crónicos, como es el caso con las LMs, la discapacidad y las consecuencias negativas aumentan de manera considerable (Burns y Ditunno, 2001; de Groot, Post, Snoek, Schuitemaker, van der Woude, 2013; Hammell, 1992; Smedema y Ebener, 2010; Vash y Crewe, 2004).

Hasta la fecha, las investigaciones sobre el US y las LMs son principalmente de tipo descriptivo y diseñadas como estudios transversales (Smedema y Ebener, 2010; Wegener, Adams y Rohe, 2012). A nuestro entender, únicamente se ha completado una revisión bibliográfica sobre este tema, pero se remonta al año 2010 y su enfoque no es exclusivamente sobre la población con LM (Smedema y Ebener, 2010).

Dados los riesgos potenciales que el US y los TUS pueden suponer en la adaptación a medio y largo plazo y en el estado de salud de los pacientes con LM (Smedema y Ebener, 2010; Wegener et al., 2012; Wright, 1960), es importante reconocer la magnitud del problema y tenerlo en cuenta como elemento clave en programas de rehabilitación (Bozzacco, 1990; Krause, 1992; Smedema y Ebener, 2010; Wegener et al., 2012). Solo esto asegurará la viabilidad del diseño de instrumentos precisos de cribado y de evaluación para diagnosticar y planificar intervenciones específicas para tratar estos temas (Hammell, 1992; Treischmann, 1988; Wright, 1960).

Por lo tanto, el objetivo de este estudio es revisar la literatura científica sobre el US y los TUS en pacientes con LM (teniendo en cuenta periodos antes y después de la lesión) y considerar su impacto sobre diferentes indicadores de salud general. Se espera que este conocimiento sea útil para fomentar más investigación en este campo y para recomendar prioridades clínicas aplicables a esta población.

\section{Métodos}

\section{Estrategia de búsqueda}

La fuente primaria de datos para esta revisión fue la base de datos electrónica MEDLINE (PubMed). También accedimos a la Biblioteca Cochrane y realizamos búsquedas en la web de Health Information Resources, www.library.nhs.uk/ default.aspx (anteriormente National Library for Health). También realizamos búsquedas en los listados de referencias de dichos artículos revisados para identificar estudios relevantes que podían haber sido omitidos. Los términos de búsqueda (únicamente en inglés) fueron spinal cord injury combinado con: substance use, substance use disorder, substance dependence, substance disorder, addiction, alcohol, tobacco, cocaine, stimulants, opioids/opiates, marihuana/marijuana, y cannabis/ cannabinoid. Resultados duplicados se eliminaron. Se omitieron guiones y abreviaturas por si esto limitase la búsqueda. Las referencias resultantes se consultaron y revisaron detalladamente. Dicha revisión sistemática se realizó acorde a las directrices PRISMA.

\section{Criterios de inclusión y exclusión}

Un único autor (CC) estableció los criterios de elegibilidad en consenso con el otro autor (PL), basándose en la información encontrada en el título, resumen, palabras clave o texto íntegro, de los artículos, cuando esto fue necesario. Los estudios se incluyeron en esta revisión si cumplían con los siguientes criterios: (1) cualquier estudio con humanos cuyo objetivo era evaluar el abuso de sustancias (tales como: alcohol, tabaco y/u otras drogas) en LM antes, durante $y$ después de rehabilitación. También se incluyeron estudios que incluían LM en la muestra, entre otros tipos de muestras. Se excluyeron estudios en idiomas distintos al inglés, o cuando únicamente se hacía referencia al uso de sustancias en la introducción o en la discusión del artículo, pero su objetivo principal no era la evaluación del uso de sustancias o el trastorno por uso de sustancias, ni se implementaban medidas específicas para ello. No se incluyeron estudios sobre fármacos prescritos, como son los medicamentos con efectos psicotrópicos (p. ej., para el tratamiento de dolor neuropático) en esta revisión. También se excluyeron comentarios, procedimientos y cartas al Editor. No se fijaron fechas límites de las publicaciones (la actualización de la búsqueda llega hasta mayo del 2014).

\section{Extracción de datos}

La extracción de datos se completó en tres fases. En la $1^{a}$ fase, un revisor realizó una búsqueda preliminar y seleccionó los estudios según los criterios de inclusión. En la $2^{\mathrm{a}}$ fase, dos revisores extrajeron los datos de manera independiente. En la $3^{\text {a }}$ fase, el resumen de la literatura fue puesto en común y analizado. Los datos de los artículos válidos se resumieron en un formulario con columnas tituladas de la siguiente forma: autor(es), año de publicación, diseño del 
estudio, muestra, herramienta(s) de evaluación y medición de uso de sustancias, otras medidas, sustancias objeto de estudio, periodo de evaluación (antes/después de lesión), prevalencia de uso de la sustancia (cuando el artículo lo detallaba) y hallazgos principales. Ambos autores (CC y PL) siguieron dicho procedimiento independientemente, y cualquier discrepancia se resolvió a través de la discusión científica.

\section{Resultados}

\section{Resultados de la búsqueda}

La búsqueda de literatura en MEDLINE resultó en 224 artículos potencialmente relevantes y relacionados con los temas pertinentes de la revisión. Se excluyeron 165 artículos que tenían el mismo título y resumen, dejando 59 artículos relevantes. Dichos artículos extraídos fueron evaluados en profundidad, según los criterios de inclusión. Cincuenta y dos de los 59 artículos previamente seleccionados cumplieron con los criterios de inclusión. De la base de datos Cochrane únicamente se extrajeron 2 artículos relevantes ya incluidos en la búsqueda en MEDLINE. Al final, 52 estudios fueron objeto de la revisión.

\section{Hallazgos generales}

El resumen de los artículos revisados se muestra en Tablas 1 y 2 .

La revisión destaca que el US y los TUS son muy frecuentes entre pacientes con LM. La mayoría de los estudios indican tasas más elevadas de US en comparación con la población general. No obstante, otros estudios no comparan la prevalencia con la población general o con otras muestras clínicas, lo que claramente limita las conclusiones. Además, la prevalencia de US y los TUS muestra una amplia variedad entre estudios. Por lo tanto, nuestros resultados señalan la necesidad de mejorar los métodos de investigación cuando se abordan estos temas.

Como indicaron algunos estudios teóricos (Smedema y Ebener, 2010), encontramos que las sustancias más estudiadas son el alcohol $(\mathrm{n}=45$, el $86.5 \%$ de los artículos incluidos) y el tabaco ( $\mathrm{n}=15$, el $28.8 \%$ de los artículos incluidos), mientras que las sustancias menos estudiadas fueron los estimulantes o sedantes (Heinemann, Doll, Armstrong, Schnoll y Yarkony, 1991; McKinley, Kolakowsky, Kreutzer, 1999). En este sentido, hay pocos estudios sobre el uso/abuso de cannabis $(\mathrm{n}=6$, el $11.5 \%$ de los artículos incluidos; Heinemann et al., 1988; 1991; Hwang, et al., 2012; Stroud, Bombardier, Dyer, Rimmele y Esselman, 2011; Turner, Bombardier y Rimmele, 2003; Young, Rintala, Rossi, Hart y Fuhrer, 1995) y de drogas ilegales $(\mathrm{n}=6$, el $11.5 \%$ de los artículos incluidos; Heinemann et al., 1991; Kolakowsky-Hayner et al., 1999; 2002; McKinley et al., 1999; Rish, Dilustro, Salazar, Schwab y Brown, 1997; Turner et al., 2003) .
En cuanto al alcohol, aunque los patrones de consumo encontrados son similares a los de la población general en términos de sexo y edad (consumo más elevado en hombres jóvenes) (Banerjea et al., 2009; Saunders y Krause, 2011; Krause, Carter y Pickelsimer, 2009), las tasas de "consumo de riesgo" son más altas para la población con LM (Tate et al., 2004; Turner et al., 2003). Más del 50\% de los pacientes lesionados muestran patrones de consumo de alcohol entre moderado-a-alto según los procedimientos de cribado y, en algunos estudios, según criterios DSM o CIE (Heinemann et al., 1991). Aunque la evidencia aún es escasa e insuficientemente concluyente, el consumo de riesgo parece estar asociado con la historia familiar de alcoholismo y con el consumo de alcohol "de riesgo" previo a la lesión (Schandler, Cohen, Vulpe y Frank, 1995; Tate, 1993).

En general, los resultados aparentemente indican tasas más altas de US (especialmente de alcohol) antes de la lesión y un agravamiento de este comportamiento después de la lesión (de Groot et al., 2013; Wegener et al., 2012). En este contexto, algunos estudios han identificado tasas elevadas de alcoholemia en el momento de sufrir la lesión, y han asociado estos resultados con la ocurrencia de la LM (Forchheimer, Cunningham, Gater y Maio, 2005; Levy et al., 2004). De manera interesante, también hay un estudio (Stroud et al., 2011) que señala que hasta el $71 \%$ de la muestra describió la aparición de la lesión como una "lección" para dejar el consumo. No obstante, es un estudio transversal sin más evidencia acerca de las tasas finales de abandono del consumo de alcohol y de las causas particulares que lo motivaron.

El tabaco es la segunda sustancia más usada por esta población clínica (Weaver et al., 2011). Como ocurre con el alcohol, hay una gran variedad en la prevalencia y el porcentaje de consumo de tabaco, según el estudio (Levy et al., 2004; Weaver et al., 2001). También cabe destacar que muchos estudios no facilitan tasas concretas sobre el uso/ consumo de tabaco, ni comparan dichos valores con la población general. Además, algunos estudios no hacen una distinción clara entre fumar tabaco o fumar cannabis, por lo que no podemos llegar a una conclusión respecto de si la prevalencia dada se refiere a tabaco, cannabis, o una combinación de ambos. En general, cuando sí se ha especificado, la prevalencia está en un rango de entre el 19-40\% de los pacientes lesionados. Aunque de forma inconstante, el uso del tabaco con frecuencia se asocia a un consumo de alcohol abusivo en esta población. Consecuentemente, esto tiene un impacto negativo sobre la salud de las personas y aumenta sus probabilidades de sufrir otras complicaciones médicas, como han demostrado algunos estudios (de Groot et al., 2013; Hwang, Chlan, Vogel y Zebracki, 2012; Krause y Saunders, 2009).

Respecto del consumo de sustancias ilegales, solo unos pocos autores han investigado este tema y de forma menos sistemática. En este sentido, los resultados se basan en procedimientos de cribado en el momento crítico de la admi- 
sión hospitalaria, sin planificación de seguimientos posteriores. Por tanto, muy pocos estudios se han completado usando procedimientos para recoger dichas medidas antes y después de sufrir las lesiones mediante un diseño longitudinal. Por ello, la prevalencia detectada puede reflejar un consumo ocasional de sustancias ilegales en lugar de un consumo continuado. Como refleja nuestro estudio (véase Tabla 2), únicamente siete estudios evaluaron US/TUS en base a criterios diagnósticos claros.

Exceptuando el cannabis, la prevalencia del consumo de drogas ilegales varía ampliamente, entre el 8-70\% de los pacientes. La prevalencia de consumo de cocaína y de psicoestimulantes varía entre el $0-14.4 \%$, mientras que el uso de opiáceos es aproximadamente del $4 \%$. Claramente, estos porcentajes son más bajos que los relacionados con el alcohol y el tabaco. Es importante señalar que esta prevalencia puede no ser completamente fiable porque varios de los estudios incluyeron el consumo de sustancias varias como sedantes, analgésicos narcóticos y barbitúricos, bajo el nombre de "sustancias psicotrópicas".

Las medidas empleadas para explorar la posible relación entre US/TUS y su impacto sobre la salud son muchas y variadas. Por lo tanto, es difícil comparar estudios y los resultados obtenibles no son concluyentes. No obstante, hay evidencia que sugiere que los pacientes considerados como consumidores de riesgo de sustancias tienen peor salud (tanto física como psicológica) (Tate, Forchheimer, Krause, Meade, y Bombardier, 2004). En este contexto, se ha relacionado un US entre moderado y severo con un mayor riesgo de problemas relacionados con el consumo de sustancias a lo largo de la vida y con peor salud (Stroud et al., 2011). Hay pocos estudios con un enfoque sobre una sustancia a la vez ( $\mathrm{n}=16$, el $30.8 \%$ de los artículos incluidos, véase Tablas 1 y 2 para más detalles). La mayoría evalúa un amplio rango de sustancias. Por lo tanto, los efectos individuales de cada uno sobre la salud de los pacientes lesionados no siempre se describen de manera específica (Banerjea et al., 2009; Smedema y Ebener, 2010; Tate et al., 2004). Es más, la prevalencia de US no siempre se facilita (Bozzacco, 1990; Krause, 1992; Njoki, Frantz y Mpofu, 2007; Perez y Pilsecker, 1994; Sweeney y Foote, 1982).

Para resumir, vale la pena mencionar que la mayoría de los estudios eran descriptivos y diseñados como estudios transversales (véase Tablas 1 y 2). Además, el periodo de evaluación de US/TUS (y el momento del mismo, antes o después de sufrir la lesión) tiene una amplia variedad entre estudios. Mientras que algunos estudios evalúan y reportan datos sobre los periodos antes y después de sufrir la lesión, la mayoría se centra en un único periodo, o el de antes o el de después. Además, sólo hay unos pocos estudios empíricos que evalúan TUS según criterios DSM o CIE (véase Tabla 2); (Banerjea et al., 2009; Findley et al., 2011; Heinemann et al., 1991; Tate et al., 2004; Turner et al., 2003). La mayoría de los artículos incluidos en esta revisión sólo dan una breve descripción de los patrones y/o prevalencia de US con respecto a una diversidad de resultados mediante el uso de instrumentos de cribado (véase Tabla 1); (Davies y McColl, 2002; Furlan y Fehlings, 2013; Hwang et al., 2012; Krause, 1992; 2004; Krause, Coker, Charliflue y Whiteneck, 2000; Tate et al., 2004). Por lo tanto, la prevalencia exacta de TUS entre la población de personas con LM no está clara. Es más, hay que mencionar que los criterios DSM y CIE han cambiado en sus versiones actualizadas, y ningún estudio empleó dichos criterios. De manera similar, únicamente dos estudios mencionan la prevalencia de una patología dual según criterios DSM (la coexistencia de TUS y trastornos mentales); (Banerjea et al., 2009; Findley et al., 2011).

Para resumir, como las revisiones teóricas anteriores han señalado, todas estas características dificultan un examen en profundidad de las relaciones a largo plazo entre US/TUD y su impacto sobre la salud (Smedema y Ebener, 2010; Wegener et al., 2012). 


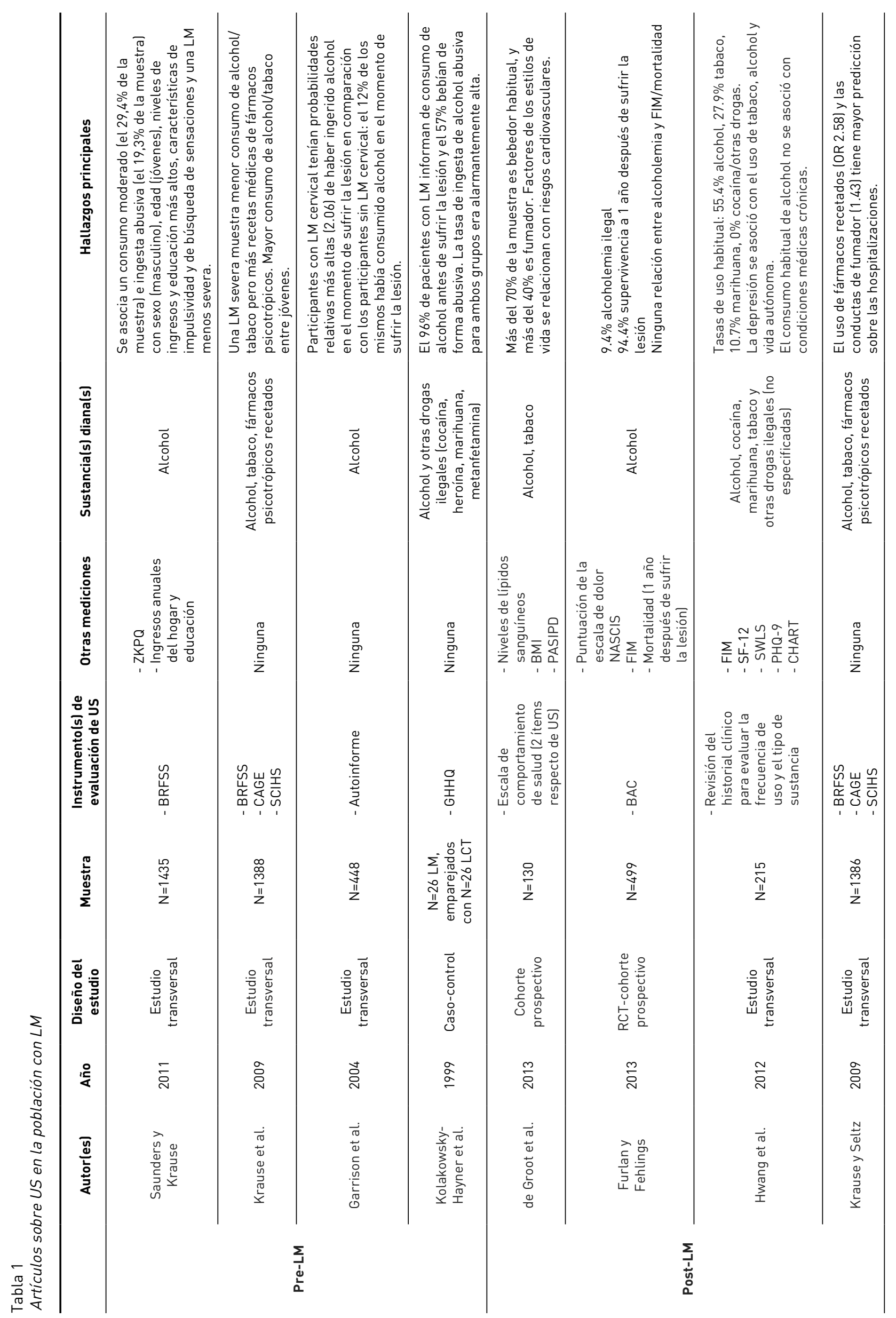




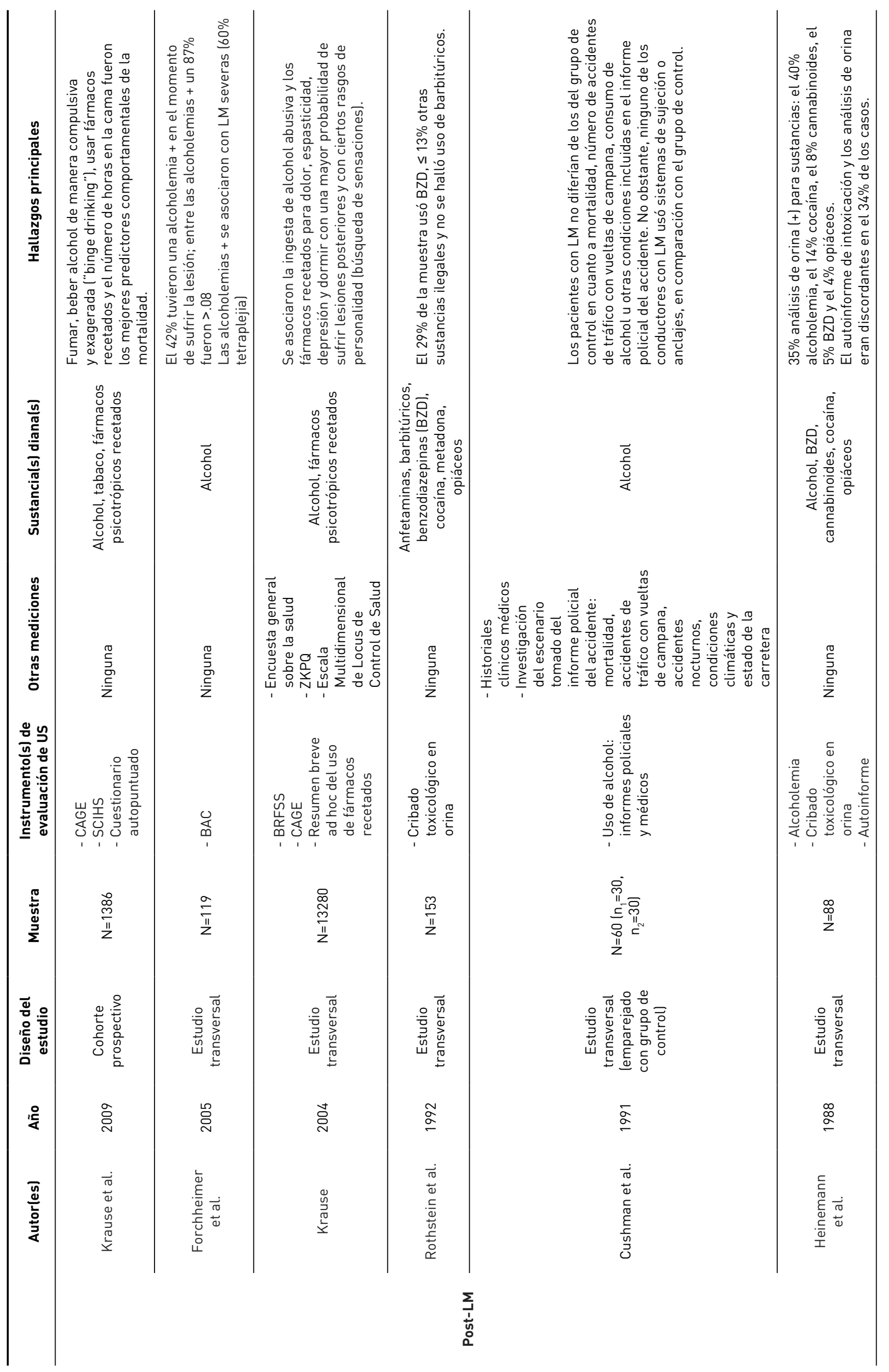




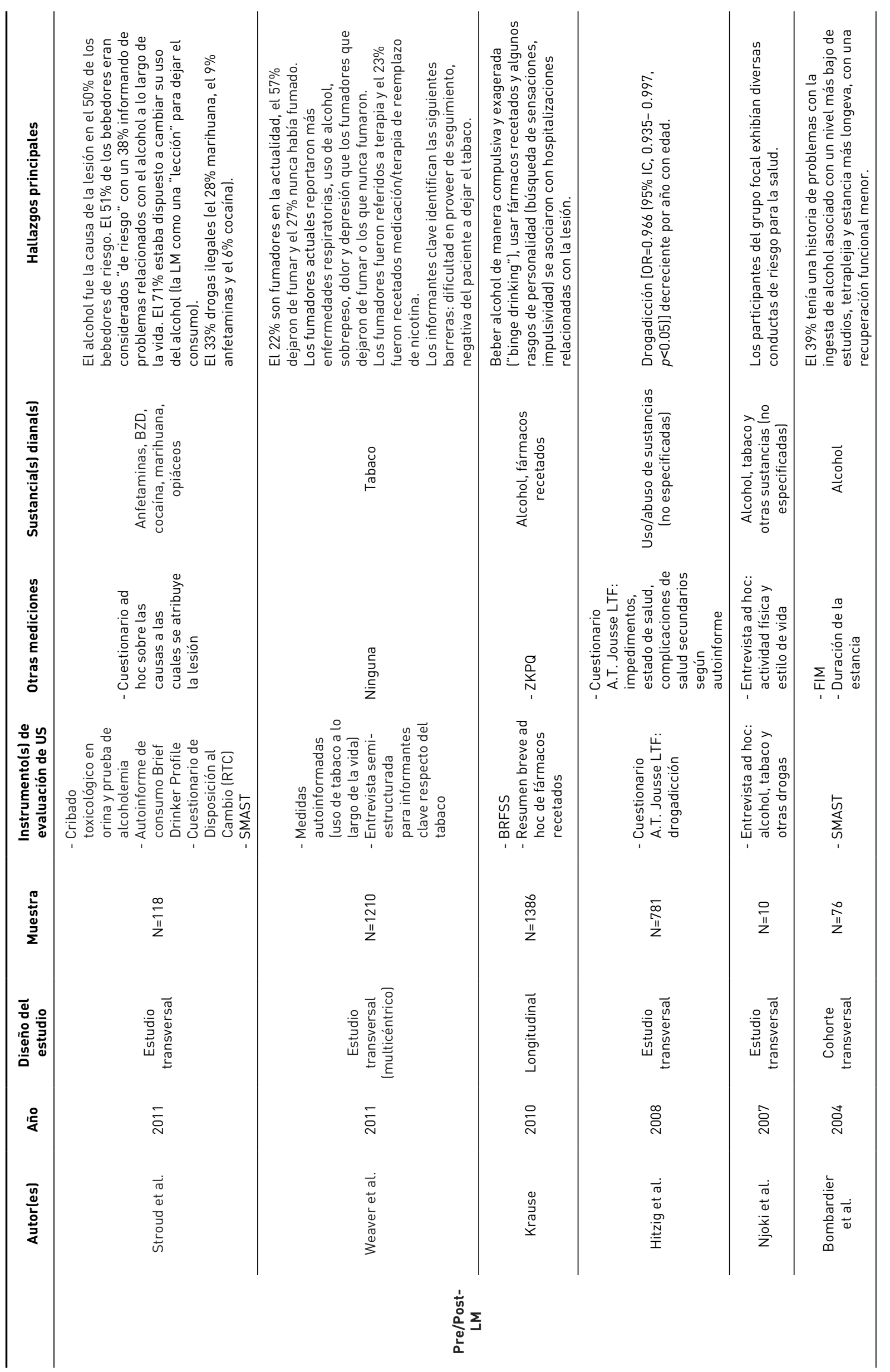




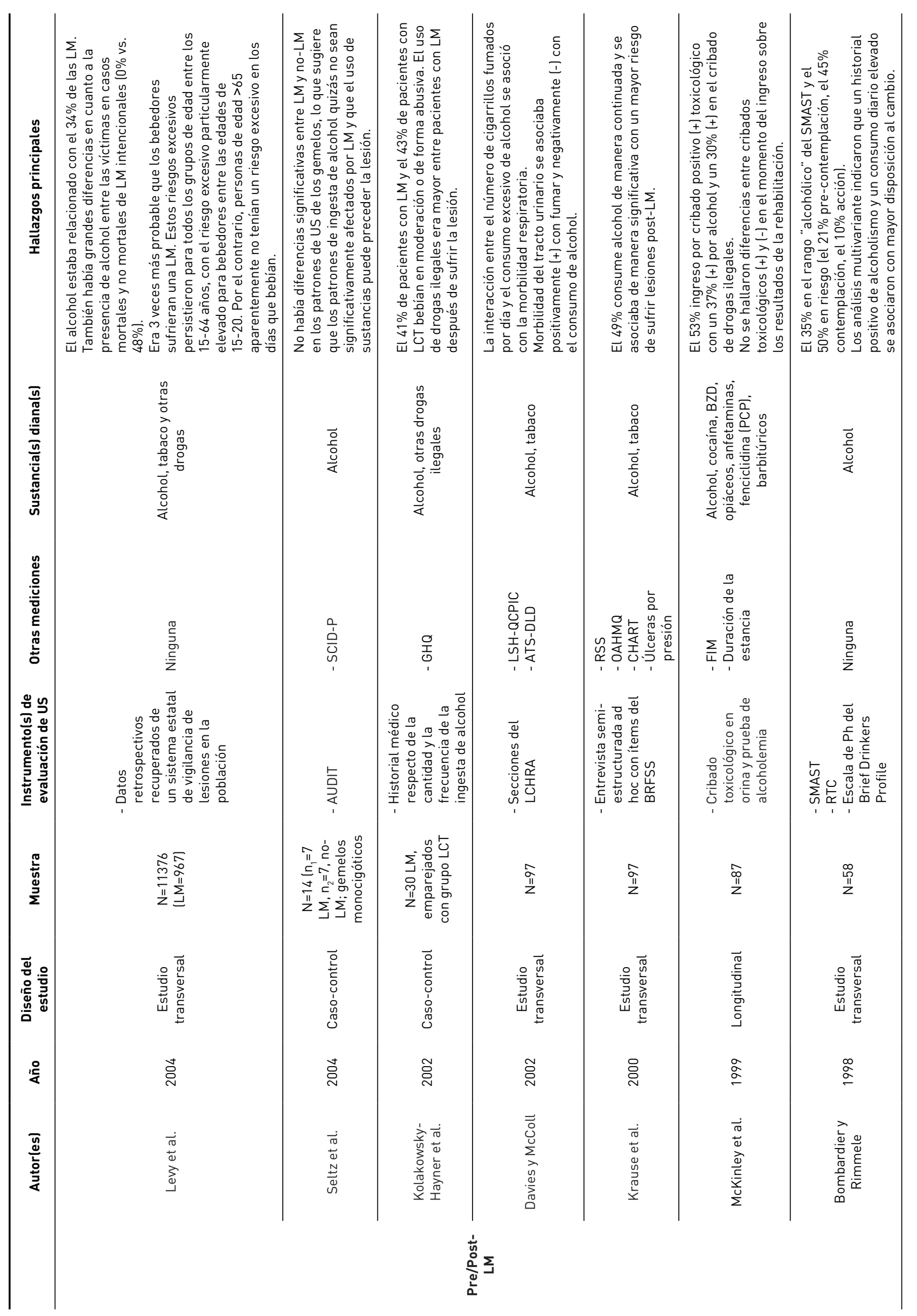




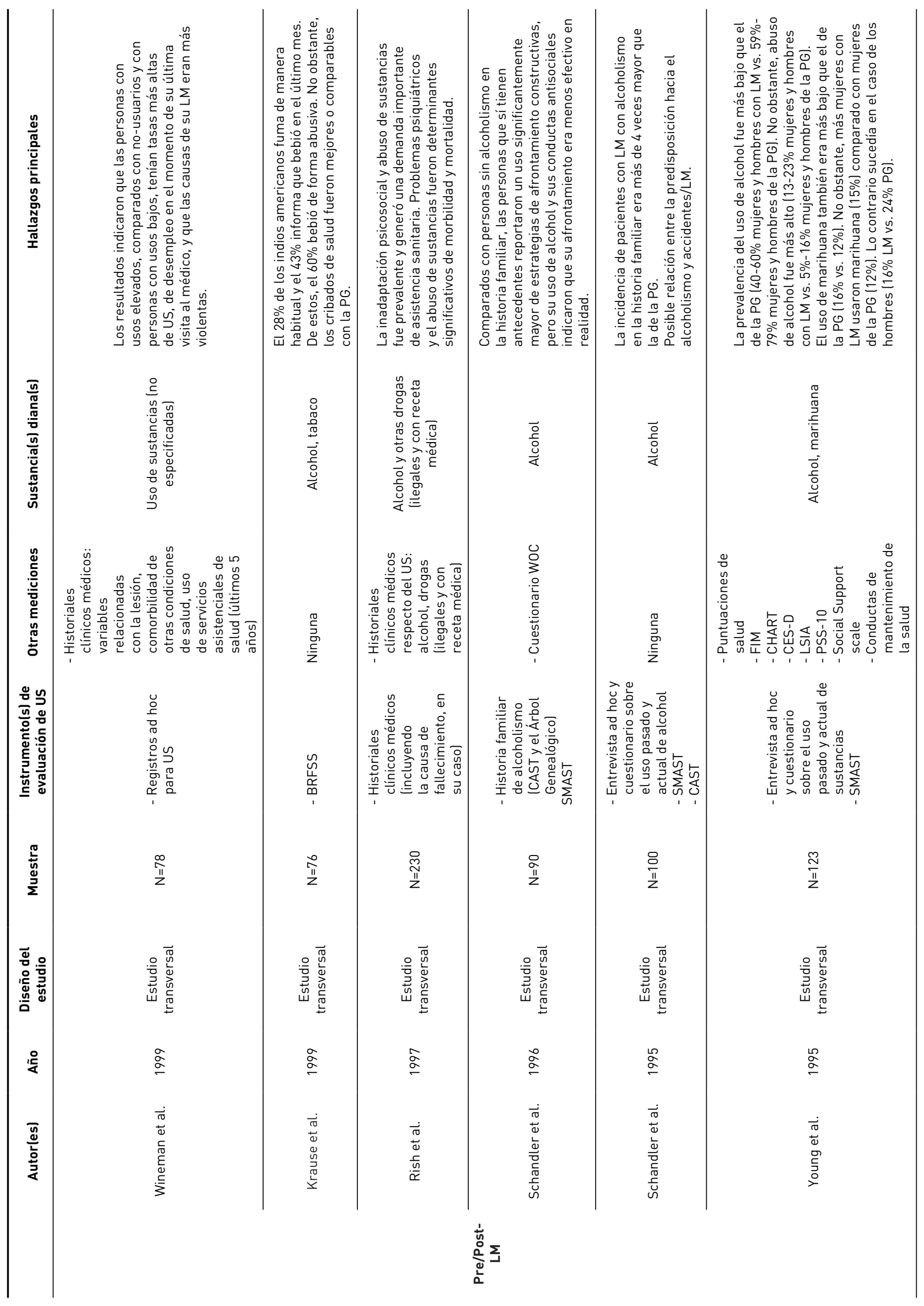




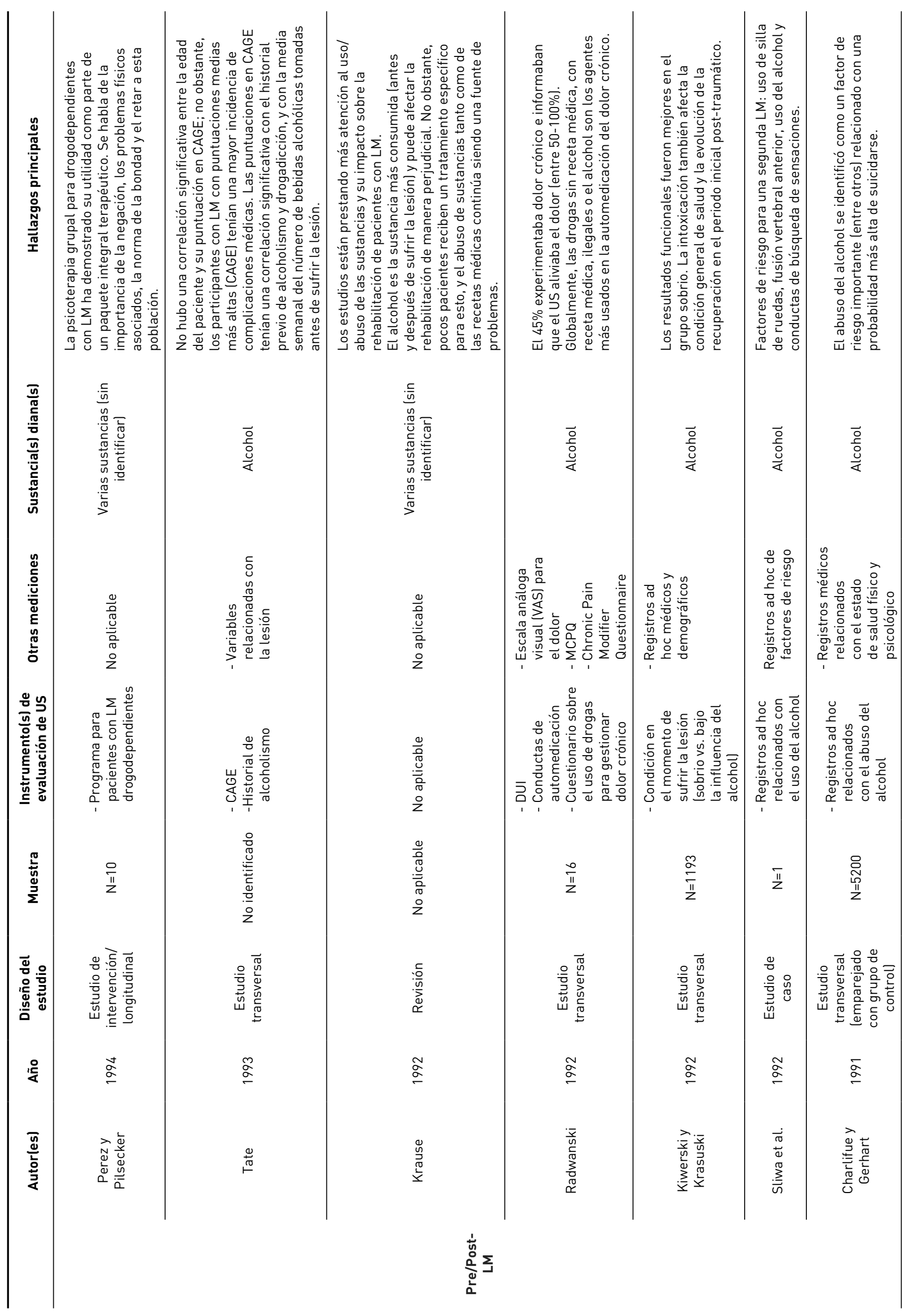




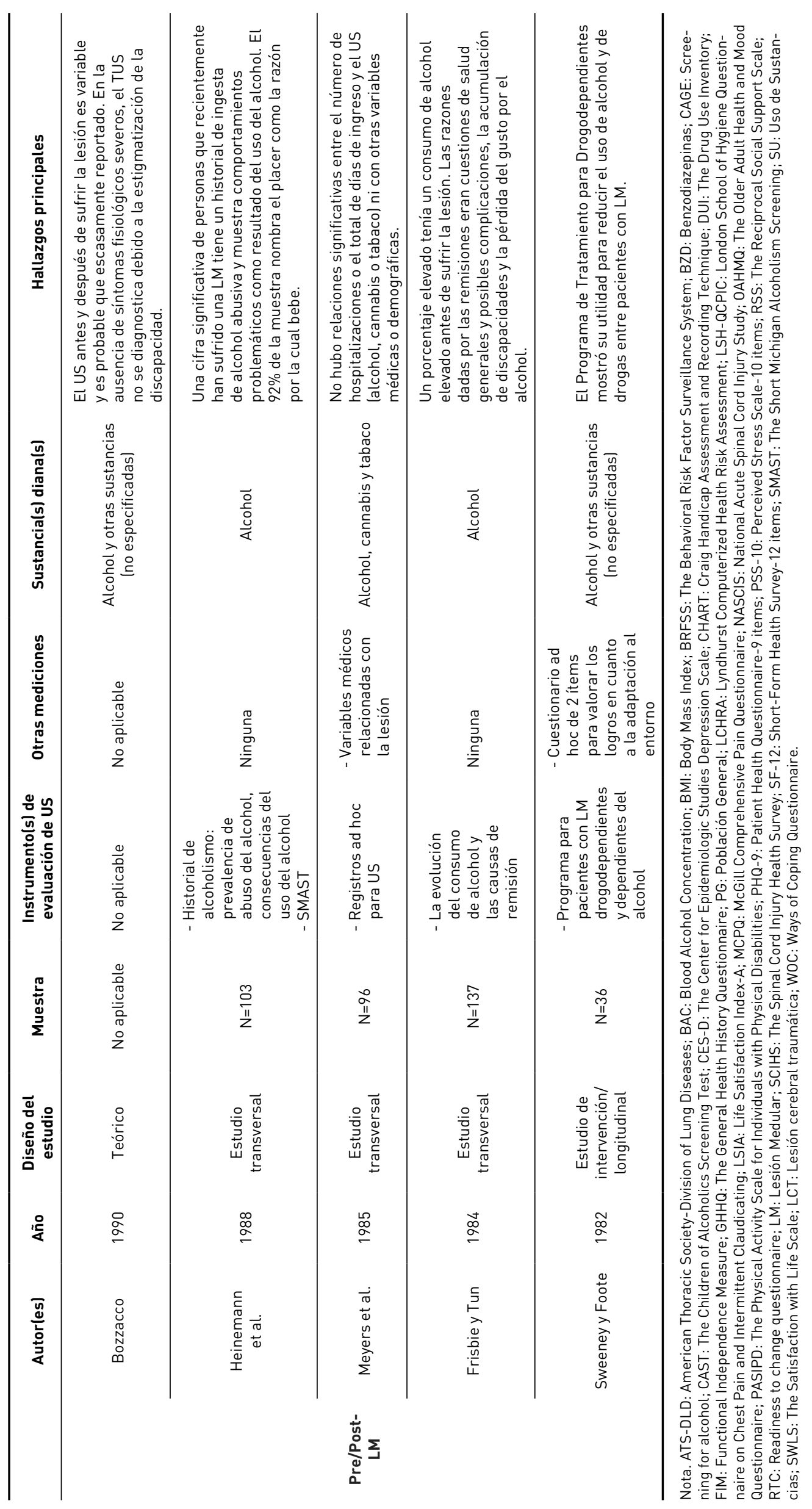




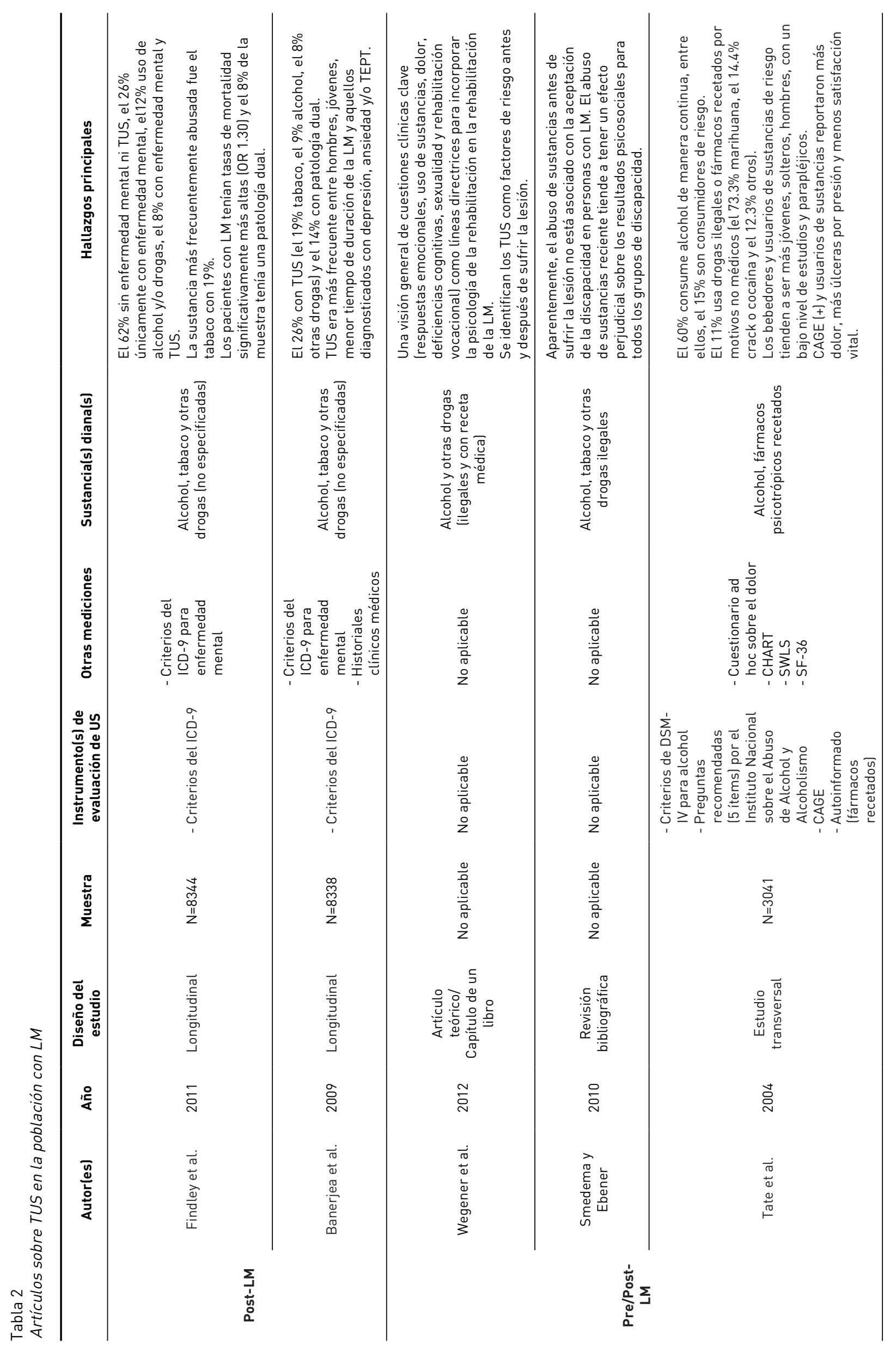




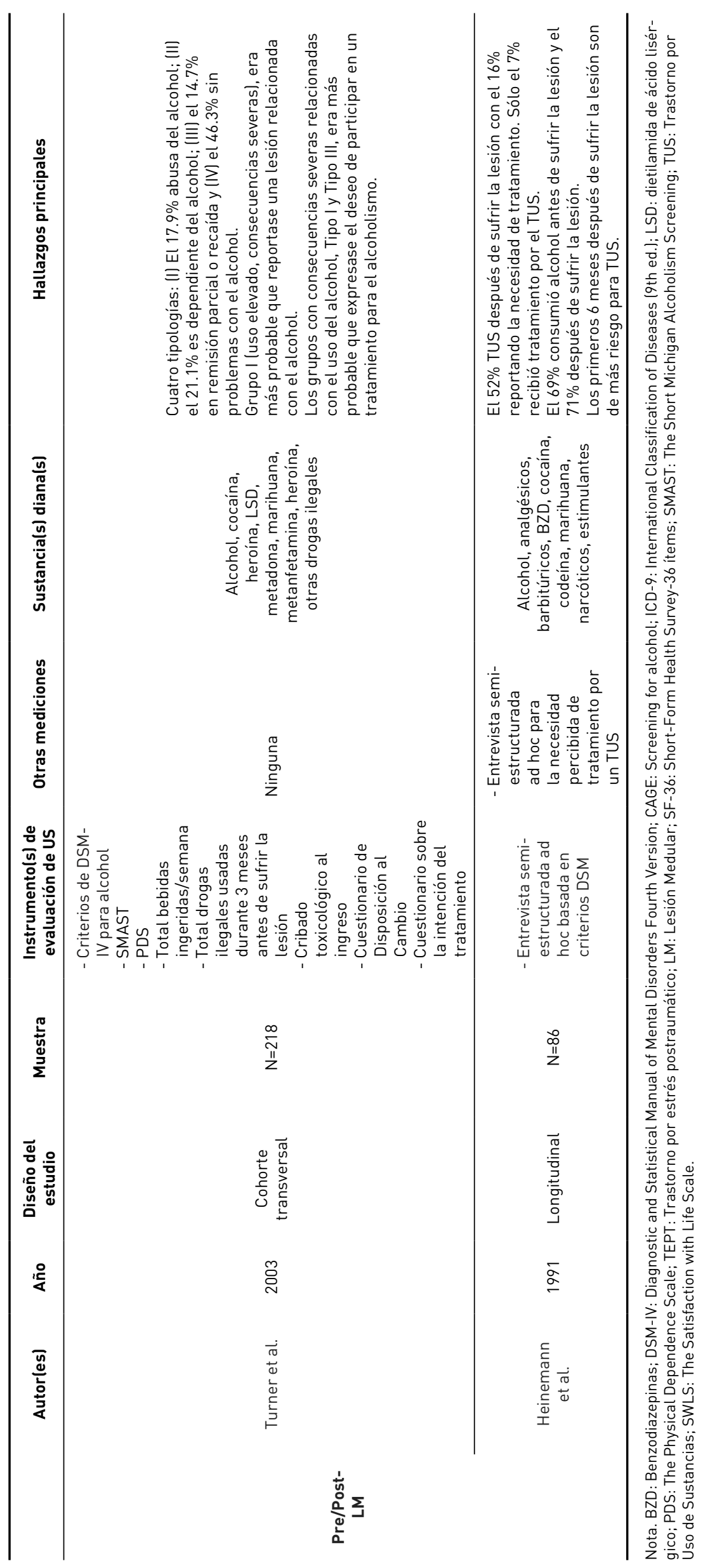




\section{Discusión}

Las personas con lesiones graves como una LM están en un nivel desproporcionadamente mayor de US y/o TUS que la población general (Kolakowsky-Hayner et al., 1999; Schandler, Cohen, y Vulpe, 1996; Schandler, Cohen, Vulpe y Frank, 1995; Smedema y Ebener, 2010; Wegener et al., 2012). Es más, como algunas investigaciones han señalado, el US también puede ser un factor importante que contribuye a sufrir una LM (Bombardier et al., 2004; Forchheimer et al., 2005; Garrison et al., 2004; Heinemann et al., 1988; Kolakowsky-Hayner et al., 1999; Krause et al., 2000; Krause, 2004; Levy et al., 2004; McKinley et al., 1999; Tate et al., 2004). Por tanto, este tema es de gran interés para profesionales sanitarios y científicos por su posible relación con la salud mental de los pacientes, la colaboración durante los procesos de rehabilitación y el funcionamiento posterior, así como en los estilos de vida elegidos después del alta (Bombardier y Rimmele, 1998; Frisbie y Tun, 1984; Njoki, Frantz y Mpofu, 2007; Post y van Leeuwen, 2012; Stroud et al., 2011).

Nuestra revisión destaca que las sustancias más frecuentemente consumidas por la población con LM son el alcohol, el tabaco y los sedantes (con y sin receta médica); también el hecho de que estos resultados están frecuentemente asociadas con el empeoramiento de la salud (p. ej., tasas más elevadas de recaídas posteriores o de complicaciones médicas) y de la salud mental (p. ej., tasas más elevadas de trastornos del estado de ánimo) (Post y van Leeuwen, 2012; Smedema y Ebener, 2010; Wegener et al., 2012). En este sentido, la prevalencia de los trastornos mentales y el uso/abuso de drogas ilegales sigue sin conocerse, aunque puede ser un motivo de preocupación para los profesionales sanitarios y habitualmente dificulta el trato con estos pacientes (Smedema y Ebener, 2010).

También encontramos que la mayoría de los estudios en este campo tienen un diseño transversal y que pocos han sido longitudinales o sobre intervenciones para prevenir o tratar el US y los TUS (Bombardier et al., 2004; Levy et al., 2004; Perez y Pilsecker, 1994; Smedema y Ebener, 2010; Sweeney y Foote, 1982; Wegener et al., 2012).

En general, hay una amplia variedad de metodologías y variables medidas entre estudios. Gran parte de la literatura se centraba en la medición de ingesta de alcohol o de alcoholemias (Furlan y Fehlings, 2013; McKinley et al., 1999; Rothstein, Levy, Fecher, Gordon, y Bauman, 1992; Stroud et al., 2011; Turner et al., 2003). Varios estudios evaluaron tabaco y otras sustancias legales (p. ej., fármacos recetados). No obstante, hay pocos estudios sobre sustancias ilegales. Es más, los periodos de evaluación son muy divergentes; algunos autores únicamente midieron el abuso de sustancias antes de sufrir la lesión, otros únicamente midieron el abuso de sustancias después de la lesión, y otros estudios evaluaban el abuso de sustancias antes de sufrir la lesión y después. Aparte, el US se midió y diagnosticó de muchas formas diferentes en cada estudio. La mayoría de los estudios usaban herramientas de cribado y no verificaron criterios DSM o CIE respecto de TUS (Banerjea et al., 2009; Findley et al., 2011; Smedema y Ebener, 2010; Tate et al., 2004). En este sentido, cabe mencionar que todos los artículos incluidos basaron sus resultados en criterios actualizados de DSM-IVTR o CIE-10 para TUS. Además, entre los estudios que emplearon criterios DSM-IV, no se menciona si aplicaron la sección sobre TUS de la Entrevista Clínica Estructurada para el DSM (SCID), que es de gran utilidad en entrevistas/evaluaciones diagnósticas. Considerando todo lo anteriormente dicho, una recomendación para estudios futuros sería el uso de la versión actualizada del DSM, el DSM-5 (American Psychiatric Association, 2013).

Esta variabilidad entre estudios respecto a la operativización de las variables principales del uso/abuso de sustancias vs. el trastorno, dificulta poder hacer comparaciones e impide llegar a conclusiones respecto de la prevalencia precisa de US y TUS entre esta población, tanto como de su posible relación con la salud física y mental.

A pesar de todo esto, se identificaron algunas tendencias importantes. Primero, se ha descrito un uso elevado de sustancias como alcohol, tabaco y/o marihuana entre los pacientes lesionados. Una hipótesis es que el abuso de sustancias puede ser empleado como un recurso de afrontamiento para disminuir la angustia y para facilitar el aislamiento de la lesión o de sus consecuencias (Bracken y Shepard, 1980; Krause, McArdle, Pickelsimer y Reed, 2009; Post y van Leeuwen, 2012). Aun así, se sabe que el abuso de las sustancias y la dependencia implica un gran riesgo para el estado de salud de pacientes con LM por las complicaciones médicas existentes y los fármacos recetados (Hitzig et al., 2008; Krause et al., 1999; 2000; 2009; Smedema y Ebener, 2010; Wegener et al., 2012). Por tanto, ha de ser adecuadamente tratado por un equipo multidisciplinar de profesionales mediante terapias de rehabilitación (Antonak y Livneh, 1991; Hammell, 1992; Vash y Crewe, 2004; Wegener et al., 2012; Wright, 1960). Como ha demostrado el estudio realizado por Stroud et al. (Stroud et al. 2011), la aparición de la lesión puede ser un momento especialmente indicado para comenzar este tipo de intervención psicoeducativa. Los comportamientos adictivos en esta población añaden otra dificultad especial e incrementan el coste de su tratamiento, implicando ingresos hospitalarios recurrentes y más citas de seguimiento médico después de sufrir la lesión (Levy et al., 2004; Krause, 2004; 2010; Schandler et al., 1995). Por lo tanto, es de vital importancia realizar un cribado de estos patrones e intervenir mediante psicoeducación, terapias grupales o intervenciones psicológicas adecuadas para reducir la comorbilidad y para facilitar una adaptación después de sufrir la lesión (Antonak y Livneh, 1991; Hammell, 1992; Smedema y Ebener, 2010; Perez y Pilsecker, 1994; Vash y Crewe, 2004; Wegener et al., 2012; Wright, 1960). Los seguimientos posteriores al alta del programa de rehabilitación son igualmente importantes para prevenir la recurrencia de proble- 
mas de dependencia (Antonak y Livneh, 1991; Hammell, 1992; Smedema y Ebener, 2010; Perez y Pilsecker, 1994; Vash y Crewe, 2004; Wegener et al., 2012; Wright, 1960). Además, los esfuerzos para intentar involucrar a la familia del lesionado, cuando es posible, podría suponer un valor añadido a las intervenciones de prevención y de rehabilitación para garantizar tasas de éxito más altas (Hammell, 1992; Smedema y Ebener, 2010; Vash y Crewe, 2004; Wegener et al., 2012; Wright, 1960).

Segundo, con frecuencia la literatura científica reporta el vínculo entre el uso de sustancias ilegales o alcohol y la aparición de LM. En la mayoría de los casos, el abuso del alcohol después de sufrir la lesión es una continuación de un patrón anterior de consumo abusivo de alcohol o de otra sustancia (Forchheimer et al., 2005; Frisbie y Tun, 1984; Kolakowsky-Hayner et al., 1999; McKinley et al., 1999; Schandler et al., 1996; Smedema y Ebener, 2010; Stroud et al., 2011; Wegener et al., 2012). Por lo tanto, el abuso de sustancias antes de la lesión parece ser un predictor importante de un abuso de sustancias después de la lesión. No obstante, la determinación del impacto de dichos patrones de abuso de sustancias sobre la salud mental de los pacientes lesionados y sobre su adaptación final es un campo merecedor de más investigación, dado que la mayoría de los estudios no eran longitudinales y los periodos de antes y después de la lesión no fueron adecuadamente estudiados respecto al US/TUS (Smedema y Ebener, 2010; Wegener et al., 2012).

\section{Conclusiones}

La literatura científica ha desvelado que muchas personas con discapacidad física o con problemas de salud crónicos muy frecuentemente tienen una discapacidad adicional, el uso (US) o abuso de sustancias (TUS) relacionados con el alcohol y/o con otras drogas psicotrópicas. Los patrones del comportamiento de US varían en función del uso antes de la aparición de la discapacidad, después de la aparición de la discapacidad, o tanto antes como después de la aparición de la discapacidad.

Aunque algunos estudios sugieren que muchas personas que viven con LM gestionan las consecuencias de su discapacidad sin niveles significativos de psicopatología, hay evidencias que apuntan a un riesgo mayor de psicopatología y de US/TUS entre estos pacientes varios años después de la aparición de la lesión. Por lo tanto, el US es un problema de salud habitual en las personas con LM. Por este motivo, los profesionales sanitarios deberían prestar especial atención a estilos de vida poco saludables y a trastornos del estado de ánimo en las citas médicas de seguimiento a corto y medio plazo, dado que se ha descrito un riesgo elevado de inadaptación y de US entre esta población.

A nuestro entender, esta es la primera revisión sistemática sobre este tema. Únicamente se ha completado una revisión bibliográfica, pero se remonta al año 2010. Aparte, no fue sistemática y su enfoque no es exclusivamente sobre la población con LM (Smedema y Ebener, 2010). Por tanto, esta revisión contribuye hacia el conocimiento y se espera que sirva como punto de partida para el diseño de otros estudios en este campo que venzan las limitaciones actuales. En este sentido, nuestros resultados han destacado la necesidad de otros estudios longitudinales que evalúen los patrones de US antes y después de la lesión, y también de estudios que evalúen la efectividad de las intervenciones que tratan dichos problemas entre pacientes con LM. Idealmente, dicho enfoque será dirigido por equipos multidisciplinares que incluyan US como la patología y el enfoque central de un programa de rehabilitación, en lugar de tratarlo como un problema secundario.

\section{Limitaciones de esta revisión}

La limitación principal de esta revisión fue la inclusión exclusiva de artículos redactados en inglés, con la consiguiente exclusión de otros estudios apropiados escritos en otros idiomas. También hay otros temas a tener en cuenta al leer nuestros resultados. La mayoría de los estudios se diseñaron con una población masculina como muestra, y sin diferenciar claramente entre pacientes parapléjicos y tetrapléjicos. Por lo tanto, la generalización de los resultados ha de hacerse con cautela. Además, no todos los estudios controlaron los posibles efectos de confusión de los fármacos prescritos en los comportamientos de US (tales como opiáceos u otros sedantes para el dolor neuropático) y algunos autores han señalado que existe un alto riesgo de TUS entre los pacientes que ya están en tratamiento con dichas sustancias.

\section{Recomendaciones para otros estudios}

La información aportada en este estudio contribuirá hacia una imagen más clara de US entre pacientes con LM. Aparte, preparará a los profesionales clínicos para reconocer los riesgos potenciales en este sentido y para discernir mejor a las personas con riesgo de US y/o TUS. Son necesarios más estudios usando diseños longitudinales para incluir resultados a largo plazo, así como del riesgo a padecer complicaciones secundarias de la salud en relación al uso de distintas sustancias específicas. Es más, sería recomendable el poder realizar un diagnóstico específico de US/TUS para drogas legales e ilegales (y no únicamente cribados) para poder comparar estudios. Esta es la única manera de que sea posible avanzar en el campo de estudios de seguimiento después de intervenir en US/TUS.

\section{Reconocimientos}

Este artículo ha sido redactado gracias a la ayuda económica aportada por Fundació La Marató de TV3 (número 
de subvención 156/U/2011) para el proyecto ESPELMA (identificador de ensayos clínicos: NCT 01889940).

\section{Conflicto de intereses}

Ninguno.

\section{Referencias}

American Psychiatric Association. (2013). Diagnostic and Statistical Manual of Mental Disorders (5th ed.). Washington, D.C.: Author.

Antonak, R. F. y Livneh, H. (1991). A Hierarchy of reactions to disability. International Journal of Rehabilitation Research, 14, 13-24. doi:1.1097/00004356-199103000-00002

Banerjea, R., Findley, P. A., Smith, B., Findley, T. y Sambamoorthi, U. (2009). Co-occurring medical and mental illness and substance use disorders among veteran clinic users with spinal cord injury patients with complexities. Spinal Cord, 47, 789-795. doi:10.1038/sc.2009.42

Bombardier, C. H. y Rimmele, C. T. (1998). Alcohol use and readiness to change after spinal cord injury. Archives of Physical Medicine and Rehabilitation, 79, 1110-1115. doi: 10.1016/S0003-9993(98)90180-0.

Bombardier, C. H., Stroud, M.W., Esselman, P.C. y Rimmele, C.T. (2004). Do preinjury alcohol problems predict poorer rehabilitation progress in persons with spinal cord injury? Archives of Physical Medicine and Rehabilitation, 85, 1488-1492. doi: 10.1016/j.apmr.2003.10.010

Bozzacco, V. (1990). Vulnerability and alcohol and substance abuse in spinal cord injury. Rehabilitation Nursing, 15, 70-72. doi:10.1002/j.2048-7940.1990.tb01436.x.

Bracken, M. B. y Shepard, M.J. (1980). Coping and adaptation following acute spinal cord injury: a theoretical analysis. Paraplegia, 18, 74-85. doi: 10.1038/sc.1980.13

Burns, A. S. y Ditunno, J. F. (2001). Establishing prognosis and maximizing functional outcomes after spinal cord injury: a review of current and future directions in rehabilitation management. Spine (Phila Pa 1976), 26, S137-145. doi:10.1097/00007632-200112151-00023.

Charliflue, S.W. y Gerhart, K.A. (1991). Behavioral and demographic predictors of suicide after traumatic spinal cord injury. Archives of Physical Medicine and Rehabilitation, 72, 488-492.

Cushman, L. A., Good, R. G. y States, J. D. (1991). Characteristics of motor vehicle accidents resulting in spinal cord injury. Accident; Analysis and Prevention, 23, 557-560. doi:10.1016/0001-4575(91)90020-6.

Davies, D. S. y McColl, M. A. (2002). Lifestyle risks for three disease outcomes in spinal cord injury. Clinical Rehabilitation, 16, 96-108. doi:10.1191/0269215502cr443oa.

de Groot, S., Post, M. W., Snoek, G. J., Schuitemaker, M. y van der Woude, L. H. (2013). Longitudinal association be- tween lifestyle and coronary heart disease risk factors among individuals with spinal cord injury. Spinal Cord, 51, 314-318.

Findley, P. A., Banerjea, R. y Sambamoorthi, U. (2011). Excess mortality associated with mental illness and substance use disorders among veteran clinic users with spinal cord injury. Disability and Rehabilitation, 33, 1608-1615. doi: 10.3109/09638288.2010.540294

Forchheimer, M., Cunningham, R. M., Gater, D. R. Jr. y Maio, R. F. (2005). The relationship of blood alcohol concentration to impairment severity in spinal cord injury. The Journal of Spinal Cord Medicine, 28, 303-307.

Frisbie, J. H. y Tun, C. G. (1984). Drinking and spinal cord injury. The Journal of American Paraplegia Society, 7, 71-73.

Furlan, J. C. y Fehlings, M. G. (2013). Blood alcohol concentration as a determinant of outcomes after traumatic spinal cord injury. European Journal of Neurology, 20, 1101-1106. doi: 10.1111/ene.12145.

Garrison, A., Clifford, K., Gleason, S. F., Tun, C. G., Brown, R. y Garshick E. (2004). Alcohol use associated with cervical spinal cord injury. The Journal of Spinal Cord Medicine, 27, 111-115.

Hammell, K. R. (1992). Psychological and sociological theories concerning adjustment to traumatic spinal cord injury: the implications for rehabilitation. Paraplegia, 30, 317-326.

Heinemann, A. W., Doll, M. D., Armstrong, K. J., Schnoll, S. y Yarkony, G. M. (1991). Substance use and receipt of treatment by persons with long-term spinal cord injuries. Archives of Physical Medicine and Rehabilitation, 72, 482-487.

Heinemann, A. W., Keen, M., Donohue, R. y Schnoll, S. Alcohol use by persons with recent spinal cord injury. (1988). Archives of Physical Medicine and Rehabilitation, 69, 619-624.

Heinemann, A.W., Schnoll, S., Brandt, M., Maltz, R. y Keen, M. (1988). Toxicology screening in acute spinal cord injury. Alcoholism, Clinical and Experimental Research, 12, 815819. doi: 10.1111/j.1530-0277.1988.tb01352.x

Hitzig, S. L., Tonack, M., Campbell, K. A., McGillivray, C. F., Boschen, K. A., Richards, K. y Craven, B. C. (2008). Secondary health complications in an aging Canadian spinal cord injury sample. American Journal of Physical Medicine and Rehabilitation, 87, 545-555. doi: 10.1097/PHM. 0b013e31817c16d6

Hwang, M., Chlan, K. M., Vogel, L. C. y Zebracki, K. (2012). Substance use in young adults with pediatric-onset spinal cord injury. Spinal Cord, 50, 497-501. doi: 10.1038/ sc. 2012.8

Kiwerski, J. E. y Krasuski, M. (1992). Influence of alcohol intake on the course and consequences of spinal cord injury. International Journal of Rehabilitation Research, 15, 240-245. doi: 10.1097/00004356-199209000-00008

Kolakowsky-Hayner, S. A., Gourley, E. V. $3^{\text {rd }}$, Kreutzer, J. S., Marwitz, J. H., Meade, M. A. y Cifu, D. X. (2002). Post-injury substance abuse among persons with brain injury and 
persons with spinal cord injury. Brain Injury, 16, 583-592. doi: $10.1080 / 02699050110119475$

Kolakowsky-Hayner, S. A., Gourley, E. V. $3^{\text {rd }}$, Kreutzer, J. S., Marwitz, J. H., Cifu, D. X. y McKinley, W. O. (1999). Pre-injury substance abuse among persons with brain injury and persons with spinal cord injury. Brain Injury, 13, 571581.

Krause, J. S. (1992). Spinal cord injury and its rehabilitation. Current Opinion in Neurology and Neurosurgery, 5, 669672.

Krause, J. S. (2004). Factors associated with risk for subsequent injuries after traumatic spinal cord injury. Archives of Physical Medicine and Rehabilitation, 85, 1503-1508. doi: 10.1016/j.apmr.2004.01.017

Krause, J. S. (2010). Risk for subsequent injuries after spinal cord injury: a 10-year longitudinal analysis. Archives of Physical Medicine and Rehabilitation, 91, 1741-1746. doi: 10.1016/j.apmr.2010.07.219

Krause, J. S., Carter, R. E. y Pickelsimer, E. (2009). Behavioral risk factors of mortality after spinal cord injury. $A r$ chives of Physical Medicine and Rehabilitation, 90, 95-101. doi: 10.1016/j.apmr.2008.07.012

Krause, J. S., Coker, J., Charliflue, S. y Whiteneck, G. G. (1999). Health behaviors among American Indians with spinal cord injury: comparison with data from the $1996 \mathrm{Be}-$ havioral Risk Factor Surveillance System. Archives of Physical Medicine and Rehabilitation, 80, 1435-1440.

Krause, J. S., Coker, J. L., Charliflue, S. y Whiteneck, G. G. (2000). Health outcomes among American Indians with spinal cord injury. Archives of Physical Medicine and Rehabilitation, 81, 924-931.

Krause, J. S., McArdle, J. J., Pickelsimer, E. y Reed, K. S. (2009). A latent variable structural path model of health behaviors after spinal cord injury. The Journal of Spinal Cord Medicine, 32, 162-174. doi: 10.1016/j.apmr.2008.08.071

Krause, J. S. y Saunders, L. L. (2009). Risk of hospitalizations after spinal cord injury: relationship with biographical, injury, educational, and behavioral factors. Spinal Cord, 47, 692-697. doi: 10.1038/sc.2009.16

Levy, D. T., Mallonee, S., Miller, T. R., Smith, G. S., Spicer, R. S., Romano, E. O. y Fisher, D. A. (2004). Alcohol involvement in burn, submersion, spinal cord, and brain injuries. Medical Science Monitor, 10, CR17-CR24. doi:

McKinley, W. O., Kolakowsky, S. A. y Kreutzer, J. S. (1999). Substance abuse, violence, and outcome after traumatic spinal cord injury. Archives of Physical Medicine and Rehabilitation, 78, 306-312.

Meyers, A. R., Feltin, M., Master, R. J., Nicastro, D., Cupples, A., Lederman, R. I. y Branch, L. G. (1985). Rehospitalization and spinal cord injury: cross-sectional survey of adults living independently. Archives of Physical Medicine and Rehabilitation, 66, 704-708.

Njoki, E., Frantz, J. y Mpofu, R. (2007). Health-promotion needs of youth with a spinal cord injury in South
Africa. Disability and Rehabilitation, 30, 465-472. doi: 10.1080/09638280600841224

Perez, M. y Pilsecker, C. (1994). Group psychotherapy with spinal cord injured substance abusers. Paraplegia, 32, 188-192. doi: 10.1038/sc.1994.34

Post, M. W. M. y van Leeuwen, C. M. C. (2012). Psychosocial issues in spinal cord injury: a review. Spinal Cord, 50, 382-389. doi: 10.1038/sc.2011.182

Radwanski, M. (1992). Self-medicating practices for managing chronic pain after spinal cord injury. Rehabilitation Nursing, 17, 312-317. doi:10.1002/j.2048-7940.1992. tb01266.x.

Rish, B. L., Dilustro, J. F., Salazar, A. M., Schwab, K. A. y Brown, H. R. (1997). Spinal cord injury: a 25-year morbidity and mortality study. Military Medicine, 162, 141-148.

Rothstein, J. L., Levy, E., Fecher, R., Gordon, S. K. y Bauman, W. A. (1992). Drug use and abuse in an urban veteran spinal cord injured population. The Journal of American Paraplegia Society, 15, 217-220.

Schandler, S. L., Cohen, M. J. y Vulpe, M. (1996). Problem solving and coping strategies in persons with spinal cord injury who have and do not have a family history of alcoholism. The Journal of Spinal Cord Medicine, 19, 78-86.

Schandler, S. L., Cohen, M. J., Vulpe, M. y Frank, S. E. (1995). Incidence and characteristics of spinal cord injured patients with a family history of alcoholism. Journal of Studies on Alcohol, 56, 522-527. doi: 10.15288/jsa.1995.56.522

Saunders, L. L. y Krause, J. S. (2011). Psychological factors affecting alcohol use after spinal cord injury. Spinal Cord, 49, 637-642. doi: 10.1038/sc.2010.160

Seltz, M., Radnitz, C. y Bauman, W. A. (2004). The relationship of spinal cord injury trauma to alcohol misuse: a study of monozygotic twins. The Journal of Spinal Cord Medicine, 27, 18-21.

Sliwa, J. A., Lim, A. C. y Roth, E. J. (1992). A second traumatic spinal cord injury: associated risk factors. Case report and review. Paraplegia, 30, 288-291. doi: 10.1038/sc.1992.71

Smedema, S. M. y Ebener, D. (2010). Substance abuse and psychosocial adaptation to physical disability: analysis of the literature and future directions. Disability and Rehabilitation, 32, 1311-1319. doi: 10.3109/09638280903514721

Stroud, M. W., Bombardier, C. H., Dyer, J. R., Rimmele, C. T. y Esselman, P. C. (2011). Preinjury alcohol and drug use among persons with spinal cord injury: implications for rehabilitation. The Journal of Spinal Cord Medicine, 34, 461472. doi: 10.1179/2045772311Y.0000000033

Sweeney, T. T. y Foote, J. E. (1982). Treatment of drug and alcohol abuse in spinal cord injury veterans. The International Journal of the Addictions, 17, 897-904. doi: 10.3109/10826088209056336

Tate, D. G. (1993). Alcohol use among spinal cord-injured patients. American Journal of Physical Medicine and Rehabilitation, 72, 192-195. doi: 10.1097/00002060-19930800000004 
Tate, D. G., Forchheimer, M. B., Krause, J. S., Meade, M. A. y Bombardier, C. H. (2004). Patterns of alcohol and substance use and abuse in persons with spinal cord injury: risk factors and correlates. Archives of Physical Medicine and Rehabilitation, 85, 1837-1847. doi: 10.1016/j.apmr.2004.02.022

Treischmann, R. B. (1988). Spinal Cord Injuries: Psychological, social and vocational rehabilitation (2nd ed). New York: Demos Medical Publishing.

Turner, A. P., Bombardier, C. H. y Rimmele, C. T. (2003). A typology of alcohol use patterns among persons with recent traumatic brain injury or spinal cord injury: implications for treatment matching. Archives of Physical Medicine and Rehabilitation, 84, 358-364. doi: 10.1053/apmr.2003.50107

Vash, C. L. y Crewe, N. M. (2004). Psychology of disability (2nd ed). New York: Springer Publishing.

Weaver, F. M., Smith, B., LaVela, S. L., Evans, C. T., Ullrich, P., Miskevics, S.,... Burns, S. P. (2011). Smoking behaviour and delivery of evidence-based care for veterans with spinal cord injuries and disorders. The Journal of Spincal Cord Medicine, 34, 35-45. doi: 10.1179/107902610X12911165975061

Wegener, S. T., Adams, L. L. y Rohe, D. (2012). Promoting optimal functioning in spinal cord injury: the role of rehabilitation psychology. Handbook of Clinical Neurology, 109, 297-314.

Wineman, N. M., Leffler, E., Radziewicz, R. M. y Sams, C. (1999). A comparative analysis of individuals with spinal cord injury who differ in unplanned utilization of health services. Spinal Cord Injury Nursing, 16, 42-47.

Wright, B.A. (1960). Physical Disability: a psychological approach. New York: Harper and Row.

Young, M. E., Rintala, D. H., Rossi, C. D., Hart, K. A. y Fuhrer, M. J. (1995). Alcohol and marijuana use in a community-based sample of persons with spinal cord injury. Archives of Physical Medicine and Rehabilitation, 76, 525-532. doi: 10.1016/S0003-9993(95)80506-0 